

\section{Method of RNA Purification from Endothelial Cells for DNA Array Experiments}

BioTechniques 32:316-320 (February 2002)

N.N. Khodarev, J. Yu, E. Nodzenski, J.S. Murley, Y. Kataoka, C.K. Brown, D.J. Grdina, and R.R. Weichselbaum

University of Chicago

Chicago, IL, USA

One of the promising applications of DNA array technology is in the design of anti-angiogenic tumor therapies through the identification of potential target genes in endothelial cells. This task requires reproducible procedures for the preparation of multiple highquality RNA samples from various in vitro and in vivo sources of endothelial cells. Unfortunately, endothelial cells possess high levels of RNase activity, which leads to the instability of RNA preparations (4). In our laboratory, we use several types of membrane-based radiolabeled DNA arrays for hybridization with cDNA prepared from different cell lines and tissue samples (Atlas ${ }^{\circledR}$ Human cDNA Expression Array; BD Biosciences Clontech, Palo Alto, CA, USA), "Known Genes" GeneFilters ${ }^{\circledR}$ GF211 (Research Genetics, Huntsville, AL, USA), and human cytokine array (R\&D Systems, Minneapolis, MN, USA). Several RNA isolation techniques were used to prepare RNA samples, including $\mathrm{TRIzOL}^{\circledR}$ Atlas Pure RNA kit, mRNA Separator kit (all from BD Biosciences Clontech), and MessageMaker $^{\mathrm{TM}}$ (Invitrogen, Carlsbad,
CA, USA) with subsequent treatment of samples with DNase I (Worthington Biochemical, Lakewood, NJ, USA). We found that these techniques led to degraded RNA or did not produce quantities of RNA from endothelial cells $\left(<0.1 \mu \mathrm{g} / 10^{6}\right.$ cells for mRNA Separator and MessageMaker) sufficient for our experiments. We require at least 15-20 $\mu \mathrm{g}$ RNA per experimental point for spectrophotometric quantitation, electrophoresis, and preparation of cDNA. Primary cultures of human endothelial cells have limited potential for growth, exhibit alterations in the expression of various proteins after the first $2-3$ passages $(2,3)$, and are expensive [a culture of $0.5 \times 10^{6}$ human umbilical vein endothelial cells (HUVEC; Clonetics, Walkersville, MD, USA) costs \$189]. Therefore, when using endothelial cells, it is important to use an RNA isolation technique that will produce sufficient quantities of highly intact RNA free from DNA contamination for use in DNA array experiments.

In our experiments with different transformed and normal human cell lines and human xenografts in athymic nude mice, we found that the use of the TRIzOL reagent led to preparations of total RNA with a sufficient yield (the average of $12.9 \mu \mathrm{g} / 10^{6}$ cells). These RNA samples had high levels of integrity (based on agarose gel electrophoresis), purity (based on $\mathrm{A}_{260} / \mathrm{A}_{280}$ ratios), and the ability to hybridize with all types of arrays tested. Figure 1A shows an example of a low background and high level of specific signals (5) for an Atlas Human cDNA Expression Array hybridized with TRIzOL-purified RNA from U87 glioblastoma cells (hybridization of the membrane in Figure 1A used the RNA shown in Figure 2A, lane 5). However, when we applied the TRIzOL technique of RNA purification to human endothelial cell lines, we found that it often produced partially or completely degraded RNA preparations (Figure 2A, lanes 1-4). Out of 59 samples tested in eight independent experiments, 38 (64\%) were partially or completely degraded. When RNA samples were intact or moderately degraded immediately following TRIzOL purification, they were easily degraded during the subsequent DNase I treatment (Figure 2B, lanes 2 and 3). Similar data were obtained for RNA samples isolated directly from the endothelium of umbilical cords. It is possible that this RNA instability is the result of high levels of RNases or nonspecific nucleases expressed by endothelial cells. Indeed, several reports have demonstrated the instability of RNA isolated from endothelial cells and a high level of endothelial RNase activity from both endogenous and exogenous sources. One example of an exogenous RNase is angiogenin, which is taken up by proliferating endothelial cells and has been shown to exhibit strong RNase activity (6). To prevent RNA degradation during the isolation procedure, several types of RNase inhibitors were used [RNase Out $^{\mathrm{TM}}$ (Invitrogen), SUPERase $\mathrm{In}^{\mathrm{TM}}$, and RNAsecure ${ }^{\mathrm{TM}}$ (both from Ambion, Austin, TX, USA)], but these did not lead to reproducible results during the RNA purification from endothelial cells.

In our previous experiments examining gene expression by RNA Northern 
Mergen

$(4 \mathrm{C})$

Circle Reader Service No. 165 
analysis, we used a column-based purification of total RNA (RNeasy ${ }^{\circledR}$ kit; Qiagen, Valencia, CA, USA) (1). The simplicity and reproducibility of this technique led us to apply it to the purification of RNA from human endothelial cells for use in our DNA array experiments. RNA samples obtained using the RNeasy kit were intact (Figure 2B, lane 4) and yielded [ $\left.{ }^{32} \mathrm{P}\right]$-labeled cDNA with a high specific activity $\left(10^{7} \mathrm{cpm} / \mu \mathrm{g}\right.$ cDNA). However, the hybridization of cDNA from these samples resulted in a high, nonspecific background on the arrays (Figure 1B). The treatment of the RNA samples with DNase I for $2 \mathrm{~h}$ at $4{ }^{\circ} \mathrm{C}$ did not lead to the complete removal of this background. Therefore, we combined this approach with a TRIzOL-based purification method because TRIzOL and subsequent phenol/chloroform extraction further purified the RNA by removing the traces of genom ic DNA and protein. This combination resulted in the purification of RNA sam ples from endothelial cell lines, HU VEC and human microvascular endothelial cells (HMEC), with high integrity, purity, and low background after hybridization with DNA arrays (Figure $1 \mathrm{C}$ and Figure 2B, lanes 5 and 6). It was important to determine whether the differences in background hybridization were connected with the RNA preparation technique or were simply the result of differences in the cell lines used, the functional status of the cells, or the hybridization conditions. To do this, we hybridized RNA samples prepared using three different techniques (TRIzOL + DNase I; RNeasy + DNase I; and RNeasy + TRIzOL + DNase I) from the same sample of cells (HUVEC) (Figure 1, D-F). For the evaluation of background, we used blank membranes provided by $\mathrm{BD}$ Biosciences Clontech. Figure 1, D and E, shows that the TRIZOL and RNeasy techniques resulted in low and high backgrounds, respectively. However, their combination resulted in RNA pure enough to remain undegraded after the DNase I treatment (Figure $2 \mathrm{~B}$, lanes 5 and 6) and in low background on the membranes (Figure 1F). Compared with the high frequency of RNA degradation that was observed when the TRIzOL method was used (64\%), our technique reduced the ratio of degraded samples to $7.5 \%$. The average yield was $11.8 \mu \mathrm{g} / 10^{6}$ cells, which is compatible with TRIzOL alone (described earlier).

The major steps of our technique are as follows: $(i)$ The detachment of en-

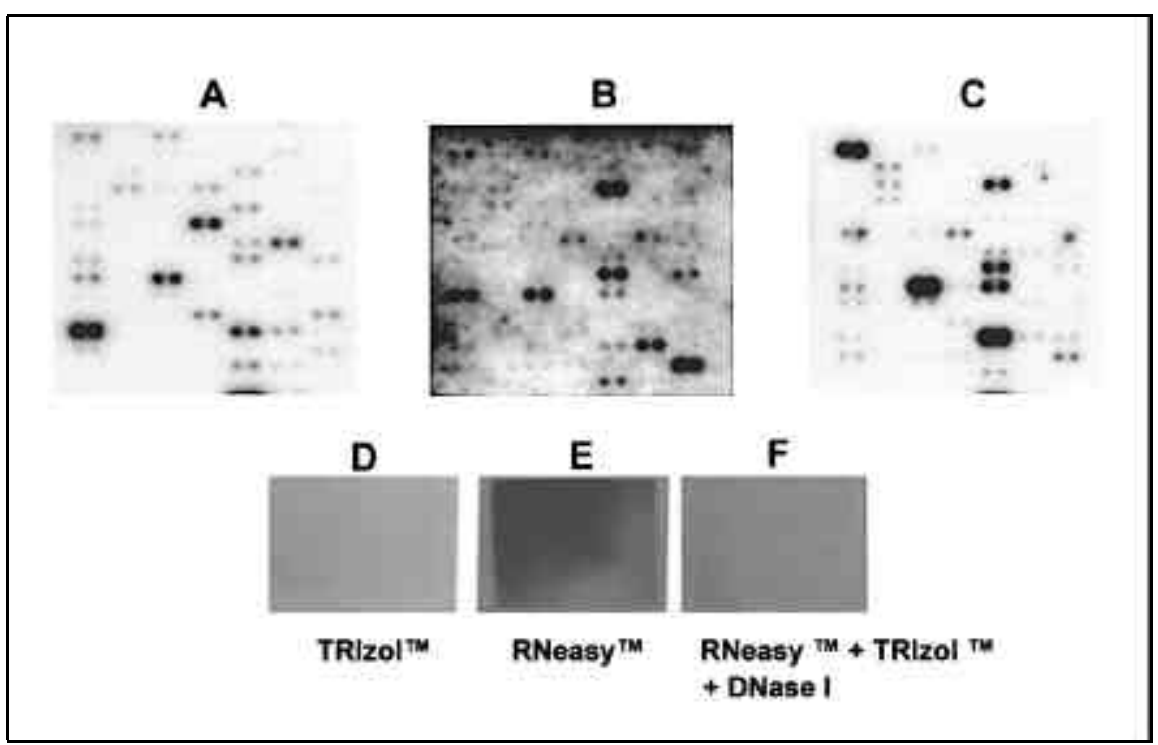

Figure 1. Effects of the RNA purification technique on hybridization with DNA arrays. The figure shows hybridizations performed with RNA samples purified from (A) U87, (B) HMEC, and (C-F) HU VEC. In panels A-C, we present the results of three independent hybridizations on one selected field of the Atlas Human cDNA Expression Arrays with RNA isolated using (A) TRIzOL, (B) RNeasy, and (C) RNeasy + TRIzoL. In panels D-F, we show the results of hybridizations using the RNA samples isolated simultaneously from the same endothelial cell line (HUVEC) using the three techniques, (D) TRIzoL, (E) RNeasy, and (F) RNeasy + TRIzoL, and hybridized with the same set of blank membranes. 
Nonlinear

(4c)

Circle Reader Service No. 167 
A

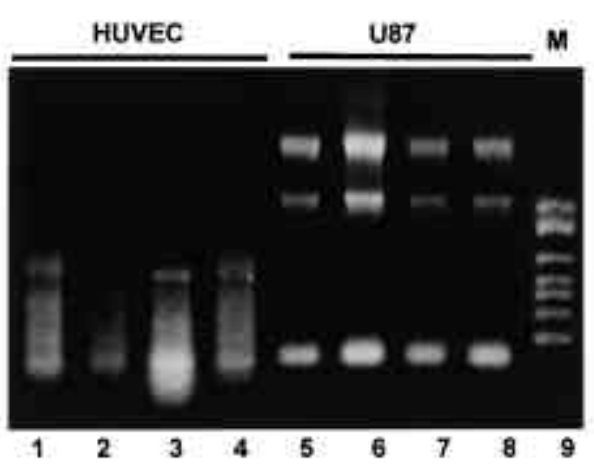

B

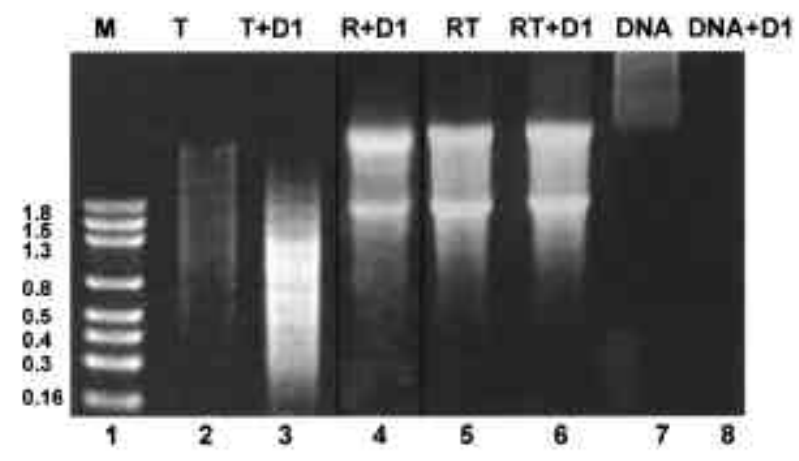

Figure 2. Degradation of HUVEC RNA is prevented by combining RNeasy with TRIzOL. (A) RNA was purified using the TRIzOL technique from human endothelial cell lines (four independent samples of each) HUVEC (lanes 1-4) and U87 glioblastoma cells (lanes 5-8). Lane 9, molecular weight standards. (B) RNA was isolated from the same cell line (HUVEC) but with different techniques. Results using TRIzOL alone and TRIzOL + DNase I are shown in lanes 2 and 3 , respectively. Note that the treatment with TRIzoL leads to the partial degradation of the RNA, and subsequent treatment with DNase I increases the level of this degradation. Lane 4 shows the results using RNeasy + DNase I. Note that the treatment with RNeasy led to RNA preparations that were not degraded under DNase I reaction conditions. Lanes 5 and 6 show the combination of RNeasy + TRIzOL and RNeasy + TRIzOL + DNase I. Lane 6 shows that this combination leads to highly pure, intact RNA. (Figure 1C presents the results of hybridization with this RNA and shows that no background is connected with this preparation.) Lanes 7 and 8 present the treatment of a plasmid DNA (lane 7) with DNase I for $2 \mathrm{~h}$ at $4^{\circ} \mathrm{C}$ (lane 8 ) as described in the text, to show that these mild conditions are adequate for the degradation of DNA. Lanes $1-4$ and 5-8 represent separate gels.

dothelial cells with trypsin/EDTA solution, cell lysis, and separation of RNA, DNA, and proteins using the RNeasy columns. This separation is based on the manufacturer's instructions, except that the final elution is done twice with $50 \mu \mathrm{L}$ RNase-free water. This step takes $1.5-2 \mathrm{~h}$ and allows the treatment of at least $10^{7}$ cells. (ii) The single-step purification of the RNA isolated in Step 1 with the TRIzOL reagent. We used an equal volume of TRIzOL and 1/5 volume of chloroform and precipitated the RNA from the water phase by adding an equal volume of $n$-isopropanol for 1 $\mathrm{h}$ at $4^{\circ} \mathrm{C}$. The RNA pellets were then dissolved in $200 \mu \mathrm{L}$ RNAsecure solution. This step takes $1.5-2 \mathrm{~h}$, depending on the number of samples. This stage may be interrupted after isopropanol addition, and the samples can be stored at $4^{\circ} \mathrm{C}$ overnight. (iii) The digestion of samples with DNase I under mild conditions (at $4{ }^{\circ} \mathrm{C}$, no exogenous $\mathrm{Mg}^{2+}$ and $\left.\mathrm{Ca}^{2+}\right)$. We recommend digestion with 5 U/sample (50-200 $\mu \mathrm{g}$ RNA) for $2 \mathrm{~h}$ at $4^{\circ} \mathrm{C}$. As shown in Figure 2B, lane 6 , this leads to the complete cleavage of DNA without the detectable degradation of RNA. (iv) Additional purification with water-saturated phenol/chloroform (1:1 mixture; water-saturated phenol was from Sigma, St. Louis,
MO, USA). The RNA was precipitated with 2.5 volumes of $95 \%$ ethanol, and the dried pellets were dissolved in 50 $\mu \mathrm{L}$ RNAsecure solution. Upon completion of these procedures, the RNA is ready to use for the synthesis of [32P]labeled cDNA for hybridization with arrays without any additional treatment. If the RNA samples are not used immediately, then they can be stored at $-80^{\circ} \mathrm{C}$ for at least six months.

\section{REFERENCES}

1.Liu, S.C., J.S. Murley, G. Woloschak, and D.J. Grdina. 1997. Repression of c-myc gene expression by the thiol and disulfide forms of the cytoprotector amifostine. Carcinogenesis 18:2457-2459.

2.Marin, V., G. Kaplanski, S. Gres, C. Farnarier, and P. Bongrand. 2001. Endothelial cell culture: protocol to obtain and cultivate human umbilical endothelial cells. J. Im munol. Methods 254:183-190.

3.McCormack, L.J., J.I. Semple, M.H. Stickland, J.H. Barrett, and P.J. Grant. 1998. The effect of number of days in culture and plasminogen activator inhibitor-1 (PAI-1) 4G/5G genotype on PAI- 1 antigen release by cultured human umbilical vein endothelial cells. Thromb. Res. 92:199-206.

4.Oribe, M., M. Shingu, and M. Nobunaga. 1986. Serum alkaline ribonuclease derived from vascular endothelial cells is raised in patients with rheumatoid vasculitis. Ann. Rheum. Dis. 45:937-940
5.Pelizzari, C.A, N.N. Khodarev, N. Gupta, D.P. Calvin, and R.R. Weichselbaum. 2000. Quantitative analysis of DNA array autoradiographs. Nucleic Acids Res. 28:4577-4581.

6.Strydom, D.J. 1998. The angiogenins. Cell. Mol. Life Sci. 54:811-824.

This work was supported in part by the National Institutes of Health/National Cancer Institute grant nos. CA71933-05 to R.R.W. and CA37435 to D.J.G.

Received 31 July 2001; accepted 29 October 2001.

Address correspondence to:

Dr. Ralph R. Weichselbaum

Department of Radiation and Cellular Oncology

5841 S. Maryland Avenue, MC9006

University of Chicago

Chicago, IL 60637, USA

e-mail:rrw@rover.uchicago.edu

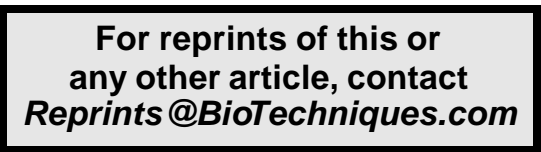




\begin{tabular}{c}
\hline Lion Bioscience \\
$(4 \mathrm{C})$
\end{tabular}

Circle Reader Service No. 168 